\title{
Effects of Triton X-100 concentration and incubation temperature on carboxyfluorescein release from multilamellar liposomes
}

\author{
M. Sila, S. Au and N. Weiner * \\ College of Pharmacy, University of Michigan, Ann Arbor, MI 48109 (U.S.A.)
}

(Received February 4th, 1986)

\begin{abstract}
Key words: Detergent-membrane interaction; Triton; Carboxyfluorescein release; Multilamellar liposome;
\end{abstract} Liposome; Temperature effect

Carboxyfluorescein is the most commonly used probe to measure the rate of release of vesicle contents. The validity of the data obtained by this method depends on obtaining an end point based on the complete release of the dye on treatment of the liposomes with a detergent, usually Triton X-100. However, Triton does not completely release entrapped carboxyfluorescein from multilamellar liposomes and the amount and rate of release of marker upon detergent treatment is a function of lipid composition of the liposome, Triton concentration and temperature and duration of detergent incubation. The fluorescence 'end point' for distearoyl-L- $\alpha$-phosphatidylcholine / cholesterol $(2: 1$, mol\%) multilamellar liposomes treated with $0.5 \%$ Triton at $22^{\circ} \mathrm{C}$ (a condition often used) is only about one-fifth the value for liposomes treated with $5 \%$ Triton at $72^{\circ} \mathrm{C}$. The conditions of treatment appear to affect the release of carboxyfluorescein from the lipid of the partially or completely disrupted liposome and the subsequent partitioning of the free dye into the aqueous phase. This effect can lead to serious errors in the interpretation of multilamellar liposome stability data. However, Triton allows complete release of entrapped dye from small unilamellar vesicles under all conditions tested.

\section{Introduction}

When liposomes are used as potential drug delivery systems, it is essential to accurately measure their mechanical integrity, e.g., the rate at which entrapped drug molecules leak out under various experimental conditions. Carboxyfluorescein, a fluorescent dye, is the most commonly used marker to assess the rates of leakage of water-soluble substances from liposomes [1,2]. The widespread use of carboxyfluorescein in this capacity is due to the sensitivity of the method and even more importantly, because the use of this marker eliminates the time-consuming process of separating free and encapsulated marker during

* To whom correspondence should be addressed. stability studies. Despite a $\mathrm{pH}$ dependency and a sensitivity to hydrophobic impurities, the carboxyfluorescein method has developed into a standard technique for these purposes [1-4]. The validity of the data obtained by this method depends on the validity of several assumptions, namely:

(1) That the entrapped carboxyfluorescein is completely self-quenched whereas there is little, if any self-quenching of the carboxyfluorescein released into the external medium. The literature suggests no problems exist with this assumption.

(2) That the treatment of the liposomes with Triton X-100 releases all of the carboxyfluorescein. It is assumed that the dye readily partitions into the external medium and does not bind to or interact with intact or disrupted liposomes [1]. 
This study presents data which clearly show the latter assumption to be invalid and a potential source of serious errors with respect to interpretations of multilamellar liposome stability.

Since the accuracy in interpreting stability data for liposomes under various experimental conditions, when expressed as the per cent of carboxyfluoresoein remaining in the liposomes, is highly dependent on the accuracy of the post-Triton end point value, a study was initiated to determine the effects of Triton concentration and the temperature and duration of Triton incubation on the end point value. Multilamellar liposomes consisting of saturated phospholipids and cholesterol were studied extensively since these lipids yield the most stable liposomes from a drug delivery point of view [17], and since the literature suggests that liposomes of this class may be somewhat resistant to Triton. Less stable multilamellar liposomes, composed primarily of egg lecithin were also studied for the sake of comparison.

\section{Materials and Methods}

Dipalmitoyl-L- $\alpha$-phosphatidylcholine, distearoyl-L- $\alpha$-phosphatidylcholine, $\mathbf{L}-\alpha$-phosphatidylcholine (derived from egg yolk), cholesterol and Triton X-100 were purchased from Sigma Chemical Co., St. Louis, MO and used without further purification. Crude carboxyfluorescein (Eastman Kodak, Rochester, NY) was purified by treatment with activated charcoal, recrystallization from ethanol and passage through a column of Sephadex LH-20 (Pharmacia, Inc., Piscataway, NJ) [8]. The resultant carboxyfluorescein showed no impurities as determined by TLC.

\section{Preparation of liposomes}

Multilamellar liposomes having the following lipid compositions were prepared:

I, Distearoyl-L- $\alpha$-phosphatidylcholine/ cholesterol $(2: 1, \mathrm{~mol} \%)$;

II, Dipalmitoyl-L- $\alpha$-phosphatidylcholine/ cholesterol $(2: 1, \mathrm{~mol} \%)$; and

III, L- $\alpha$-phosphatidylcholine.

Lipid samples ( $30 \mu$ moles) dissolved in chloroform were dried and residual solvent was removed in vacuo. The dried lipid was suspended by vortexing in $50 \mathrm{mM}$ Hepes buffer containing $200 \mathrm{mM}$
$\mathrm{NaCl}$ and $100 \mathrm{mM}$ carboxyfluorescein, final $\mathrm{pH}$ 7.0. The resultant phospholipid concentration was about $30 \mathrm{mM}$. Empty liposomes were also prepared by a similar procedure but contained no carboxyfluorescein. The lipid mixtures were incubated for one hour with occasional vortexing at a temperature above the phase transition temperature of the phospholipid component of the liposome; Lipid mixture III was incubated at $22^{\circ} \mathrm{C}$. The dispersions were then sonicated for one min in a bath-type sonicator (Branson Cleaning Equipment, Shelton, CT) at the same temperature as the incubation. Free carboxyfluorescein was removed by passage of the dispersion through a $1 \times 45$ column of Sephadex G-50 (Pharmacia, Inc., Piscataway, NJ) or by centrifugation, three times, for $10 \mathrm{~min}$ at $10000 \mathrm{rpm} \mathrm{[17].} \mathrm{Removal} \mathrm{of} \mathrm{free}$ carboxyfluorescein resulted in 5- to 20 -fold dilution of the dispersions. Also, the centrifuged pellets were reconstituted with varying volumes of buffer to yield dispersions having phospholipid concentrations ranging from about 1 to $30 \mathrm{mM}$.

Small unilamellar vesicles composed of distearoyl-L- $\alpha$-phosphatidylcholine/cholesterol (2 : 1 , mol\%) and containing entrapped carboxyfluorescein were prepared by sonication of the multilamellar liposomes for two hours. Large unilamellar vesicles of similar composition were prepared by the method described by Magin and Weinstein [19]. Free carboxyfluorescein was removed by passage of the dispersion through a $1 \times 45 \mathrm{~cm}$ column of Sephadex G-50.

\section{Fluorescence measurement}

All fluorescence measurements were made with a Perkin-elmer, Model LS-5 spectrofluorometer (excitation and emission slit widths were $10 \mathrm{~nm}$ and $3 \mathrm{~nm}$, respectively). Carboxyfluorescein was excited at $490 \mathrm{~nm}$ and emission was read at 520 $\mathrm{nm}$. Fluorescence is self-quenched at high concentrations of carboxyfluorescein due to dye-dye interactions [18]. A standard curve of fluorescence vs. carboxyfluorescein concentration confirmed that entrapped carboxyfluorescein is completely self-quenched and the carboxyfluorescein freed by Triton treatment shows no or minimal selfquenching.

In order to determine if Triton X-100 or the liposomes themselves interfere with the fluores- 
cence of carboxyfluorescein, various concentrations of Triton $(0.5 \%$ to $5.0 \%)$ and various concentrations of distearoyl-L- $\alpha$-phosphatidylcholine/cholesterol and $\mathrm{L}-\alpha$-phosphatidylcholine empty liposomes were added to carboxyfluorescein solutions. The mixtures were incubated for 90 min at a temperature above the phase transition temperature of the phospholipid component of the liposome. The presence of detergent or liposomes had no affect on the fluorescence of carboxyfluorescein.

\section{Effect of Triton concentration and incubation tem- perature on fluorescence}

$40 \mu 1$ of liposomal dispersion (containing 50 nmoles to $1.2 \mu$ moles lipid), with entrapped carboxyfluorescein were diluted with Hepes buffer. The samples were then incubated in various concentrations of Triton X-100 at different temperatures for up to $90 \mathrm{~min}$. The final volumes of the samples varied between 3.0 and $6.0 \mathrm{ml}$. The distearoyl-L- $\alpha$-phosphatidylcholine/cholesterol liposomes were incubated in $0.5 \%$ and $3 \%$ Triton at $22^{\circ} \mathrm{C}$ and $72^{\circ} \mathrm{C}$ and in $5 \%$ Triton at $72^{\circ} \mathrm{C}$; the dipalmitoyl-L- $\alpha$-phosphatidylcholine / cholesterol liposomes were incubated in $0.5 \%$ and $3 \%$ Triton at $53^{\circ} \mathrm{C}$; and the $\mathrm{L}-\alpha$-phosphatidylcholine liposomes were incubated in $0.5 \%$ and $3 \%$ Triton at $22^{\circ} \mathrm{C}$. In some experiments, distearoyl-L- $\alpha$-phosphatidylcholine containing liposomes, after $10 \mathrm{~min}$ of Triton incubation, were sonicated above or below the phase transition temperature of the lipid for 2, 3, 5 or $10 \mathrm{~min}$. Fluorescence was determined at various time intervals for each of the experimental procedures described.

Effect of Triton concentration on release of entrapped sucrose

In order to determine if the effects of Triton concentration on fluorescence are caused by the inability of the detergent to disrupt the liposomes completely, $\left[{ }^{14} \mathrm{C}\right]$ sucrose (New England Nuclear, Boston, MA) entrapped in distearoyl-L- $\alpha$-phosphatidylcholine/cholesterol multilamellar liposomes were treated with $0.5 \%$ and $3.0 \%$ Triton for $15 \mathrm{~min}$ at $72^{\circ} \mathrm{C}$. The samples were then centrifuged three times at $10000 \mathrm{rpm}$ for $10 \mathrm{~min}$ and the supernatants and pellet were assayed by liquid scintillation counting (Beckman Instruments, Model LS-9000) as described previously [17].

\section{Results}

Effect of Triton concentration and incubation temperature on fluorescence

The effects of Triton X-100 concentration and incubation temperature on distearoyl-L- $\alpha$-phosphatidylcholine/ cholesterol multilamellar liposomes are shown in Fig. 1. The fluorescence values are depicted as relative values for the sake of clarity (absolute values are dependent on the initial amount of carboxyfluorescein entrapped). The experimental condition yielding the highest fluorescence value for a given batch of liposomes (incubation with $5 \%$ Triton at $72^{\circ} \mathrm{C}$ for $90 \mathrm{~min}$ ) was assigned a value of 100 . It is conceivable that even higher absolute values can be obtained under more strenuous experimental conditions. The relative values of the four liposome batches tested agreed to within about $2-3 \%$. Since these batches represented 10 - to 20 -fold differences in lipid concentration, the effects of Triton concentration and incubation temperature appear to be independent of lipid concentration.

The post-Triton end point depends strongly on the incubation temperature and Triton concentration. At an incubation temperature below the phase transition temperature of distearoyl-L- $\alpha$-phosphatidylcholine $\left(22^{\circ} \mathrm{C}\right)$, the fluorescence was considerably more time dependent than at an incubation temperature above the phase transition temperature $\left(72^{\circ} \mathrm{C}\right)$. It is indeed striking that the fluores-

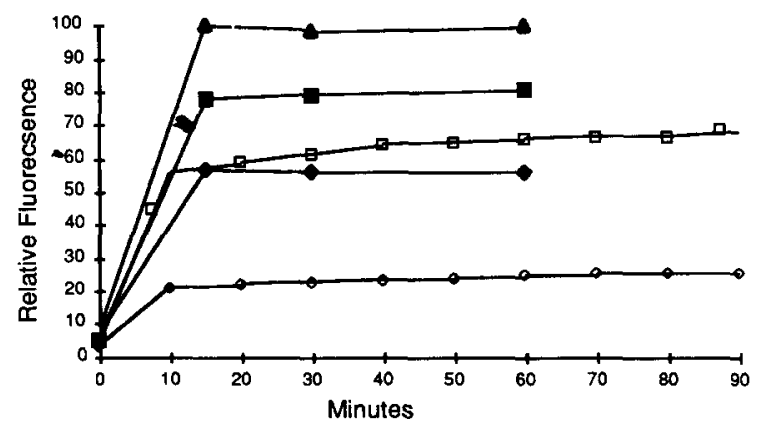

Fig. 1. Relative fluorescence as a function of the time of incubation for distearoyl-L- $\alpha$-phosphatidylcholine/cholesterol ( $2: 1$, mol\%) liposomes containing entrapped carboxyfluorescein and incubated with various concentrations of Triton X-100 at different temperatures. Key: $(\diamond) 0.5 \%$ Triton at $22^{\circ} \mathrm{C}$; $(\square)$ $3.0 \%$ Triton at $22^{\circ} \mathrm{C}$; (४) $0.5 \%$ Triton at $72^{\circ} \mathrm{C}$; (D) $3.0 \%$ Triton at $72^{\circ} \mathrm{C}$; (4) $5.0 \%$ Triton at $72^{\circ} \mathrm{C}$. 
cence 'end point' for liposomes treated with $0.5 \%$ Triton at $22^{\circ} \mathrm{C}$ (a condition often used) is only about one-fifth the value for liposomes treated with $5 \%$ Triton at $72^{\circ} \mathrm{C}$. Sonication of the samples for various periods of time up to $20 \mathrm{~min}$ (after 10 min of incubation) resulted in increased values of fluorescence only for samples incubated at temperatures below the phase transition temperature. The effect of Triton concentration on dipalmitoylL- $\alpha$-phosphatidylcholine/ cholesterol liposomes $\left(0.5 \%\right.$ and $3.0 \%$ Triton incubated at $\left.53^{\circ} \mathrm{C}\right)$ was similar to that of the distearoyl-L- $\alpha$-phosphatidylcholine/cholesterol liposomes. In the former case the higher Triton concentration treatment resulted in a fluorescence value 1.3-times greater than for the lower Triton concentration treatment, whereas the value for the latter case is 1.4.

The effects of Triton X-100 concentation on L- $\alpha$-phosphatidylcholine multilamellar liposomes are shown in Fig. 2. Again the fluorescence values are depicted as relative values for the sake of clarity. Even for the case of the relatively unstable egg lecithin liposomes, treatment with $3 \%$ Triton results in an 'end point' about $25 \%$ higher than treatment with $0.5 \%$ Triton.

Treatment of distearoyl-L- $\alpha$-phosphatidylcholine/cholesterol small unilamellar vesicles and large unilamellar vesicles with Triton resulted in complete release of the dye within $10 \mathrm{~min}$ for Triton concentrations of $0.5 \%$ and $1 \%$ at both $20^{\circ} \mathrm{C}$ and $72^{\circ} \mathrm{C}$.

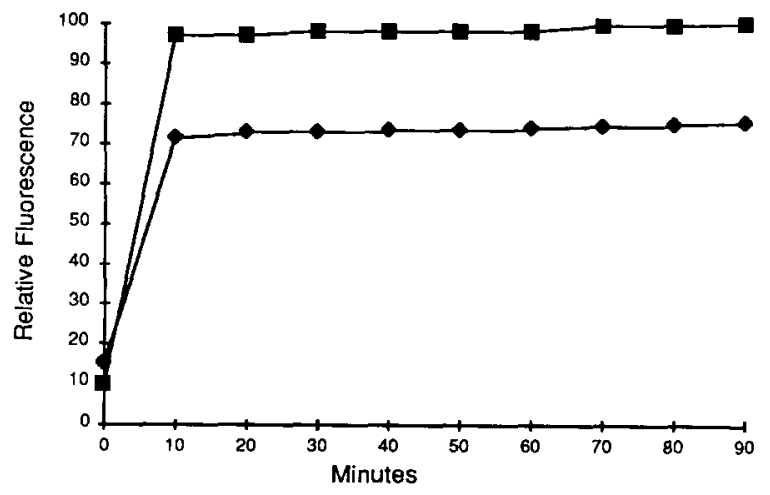

Fig. 2. Relative fluorescence as a functio of the time of incubation for L- $\alpha$-phosphatidylcholine (egg lecithin) liposomes containing entrapped carboxyfluorescein and incubated with Triton X-100 at $22^{\circ} \mathrm{C}$. Key: (४) $0.5 \%$ Triton; (D) $3.0 \%$ Triton.
Effect of Triton concentration on release of entrapped sucrose

Release of entrapped $\left[{ }^{14} \mathrm{C}\right]$ sucrose by $0.5 \%$ and $3.0 \%$ Triton, as determined by liquid scintillation counting, was $96 \%$ and $98 \%$, respectively. Thus, the effect of Triton on fluorescence readings, under the experimental conditions described, does not appear to be due to the inability of Triton (even at a concentration of $0.5 \%$ ) to disrupt the liposomes sufficiently so as to allow release of water-soluble marker into the aqueous phase.

\section{Discussion}

Stability of liposomes (often expressed as the per cent of carboxyfluorescein remaining in the liposomes) is usually determined by measuring the fluorescence of a diluted liposomal dispersion before and after treatment with Triton X-100. This detergent treatment is assumed to free all of the carboxyfluorescein which then partitions totally into the aqueous phase (assumption 2). There is great variability in the methods used to determine the post-Triton X-100 fluorescence end point [1-14]. For example, the final concentration of Triton used varies from $0.1 \%$ to $2.5 \%$ and the temperature of incubation with Triton varies from those below to those above the phase transition temperature of the phospholipid component of the liposome. Furthermore, the concentration of Triton used and the temperature and duration of incubation are often not reported.

There are a number of reports in the literature which indicates that liposomal treatment with Triton does not completely release entrapped marker and the amount and rate of release of marker upon detergent treatment is a function of lipid composition of the liposome, Triton concentration and temperature and duration of detergent incubation. Parente and Lentz [7] reported that it was necessary to record fluorescence measurements for two hours to ensure complete release of marker after Triton treatment. Senior and Gregoriadis [20] reported that higher temperatures (e.g., boiling) lead to increased carboxyfluorescein release from multilamellar liposomes. Inoue and Kitagawa [13], in an extensive study of the sensitivities of various glucose encapsulated liposomes to Triton, concluded that lipid membranes in the 
gel state are more sensitive to detergent than membranes in the liquid-crystalline state. They also reported that the rate and extent of release of marker were dependent on a number of variables such as lipid composition of the bilayer and the type of liposome studied (multilamellar vs. small unilamellar). Kinsky et al. [15] reported that liposomes composed primarily of sphingomyelin showed resistance to the action of Triton whereas liposomes composed primarily of egg lecithin did not. Dipalmitoyl-L- $\alpha$-phosphatidylcholine liposomes with $50 \mathrm{~mol} \%$ of cholesterol also resisted the detergent [16].

It is generally accepted that disruption of liposomes by detergents, even at concentrations of $0.5 \%$, is straightforward, resulting in the instantaneous destabilization of the liposome with total liberation and subsequent partition into the aqueous phase of the water-soluble marker (see for example, Ref. 2). The results of this study show that when carboxyfluorescein is used as the aqueous marker, one can not safely make this assumption for multilamellar liposomes.

For both 'stable' liposomes (composed of saturated phospholipids and cholesterol) and 'unstable' liposomes (composed of egg lecithin), the post-Triton fluorescent end point is very dependent on the concentration of Triton used to disrupt the liposome and slightly dependent on the time of incubation. Also, for 'stable' liposomes, the end point is very dependent on incubation temperature.

The reason for these effects is not clear but the results can not be explained solely on the basis of the degre of 'leakiness' of the liposome upon treatment by Triton. Since sucrose is completely liberated even at low Triton concentrations, the conditions of treatment (Triton concentration and incubation temperature) appear to affect the release of carboxyfluorescein from the lipid of the partially or completely disrupted liposome and the subsequent partitioning of the free dye into the aqueous phase.

Irrespective of the reason for these effects, there are important practical implications with respect to the use of carboxyfluorescein in evaluating multilamellar liposome stability. An example using the data in Fig. 1 can illustrate this potential problem. If a stability data point results in a relative fluorescence reading of 10 , the total percentage of leakage calculated will be dependent on the condition of the post-Triton end point. If the end point was calculated using $0.5 \%$ Triton incubated at $22^{\circ} \mathrm{C}$ (relative fluorescence $=20$ ), the leakage will be estimated at about 50\%. If, however, the end point was calculated using 5.0\% Triton incubated at $72^{\circ} \mathrm{C}$ (relative fluorescence $=$ 100 ), the leakage will be estimated at about $10 \%$.

Clearly, differences of this magnitude can lead to serious errors in the determination of stability data. Standardization of the procedure used to obtain the post-Triton end point is needed to obtain reliable 'relative' stability data such as a comparison of the effects of storage conditions on stability or batch-to-batch variability. However, even such standardization will not yield accurate values of the absolute amount of material released since it is possible that higher Triton concentrations or higher incubation temperatures could result in even further release of dye. Furthermore, since the lipid composition of the liposome could affect the post-Triton end point, the reliability of the fluorescence method to compare the stability of multilamellar liposomes of different compositions would be suspect.

\section{References}

1 Weinstein, J., Ralston, N.E., Leserman, L.D., Klausner, R.D., Dragsten, P., Henkart, P. and Blumenthal, R. (1984) in Liposome Technology (Gregoriadis, G., ed.), pp. 183-204, CRC Press, Boca Raton, FL

2 Lelkes, P.I. (1984) in Liposome Technology (Gregoriadis, G., ed.), pp. 225-246, CRC Press, Boca Raton, FL

3 Düzgüneş, N., Wilschut, J., Hong, K., Fraley, R., Perry, C., Friend, D.S., James, T.L. and Papahadjopoulos, D. (1983) Biochim. Biophys. Acta 732, 289-299

4 Barbet, J., Machy, P. and Leserman, L.D. (1981) J. Supramol. Struct. Cell. Biochem. 16, 243-258

5 Barbet, J., Machy, P., Truneh, A. and Leserman, L.D. (1984) Biochim. Biophys. Acta 772, 347-356

6 Crommelin, D.J.A. and Van Bommel, E.M.G. (1984) Pharm. Res. 159-163

7 Parente, R.A. and Lentz, B.R. (1984) Biochemistry 23, 2353-2362

8 Ralston, E., Hjelmeland, L.M., Klausner, R.D., Weinstein, J.N. and Blumenthal, R. (1981) Biochim. Biophys. Acta 649, 133-137

9 Klausner, R.D., Kumar, N., Weinstein, J.N., Blumenthal, R. and Flavin, M. (1981) J. Biol. Chem. 256, 5879-5885

10 Kercret, H., Chiovetti, R., Jr., Fountain, M.W. and Segrest, J.P. (1983) Biochim. Biophys. Acta 733, 65-74 
11 Okimasu, E., Sasaki, J. and Utsumi, K. (1984) FEBS Lett. $168,43-48$

12 Wilschut, J.C., Regts, J. and Scherphof, G. (1979) FEBS Lett. 98, 181-185

13 Inoue, K. and Kitagawa, T. (1976) Biochim. Biophys. Acta $426,1-16$

14 Rowland, R.N. and Woodley, J.F. (1981) Biosci. Rep. 1, 345-352

15 Kinsky, S.C., Haxby, J.A., Zopf, D.A., Alving, C.R. and Kinsky, C.B. (1969) Biochemistry 8, 4149-4158

16 Inoue, K. (1974) Biochim. Biophys. Acta 139, 390-402
17 Schwinke, D.L., Ganesan, M.G. and Weiner, N.D. (1984) Int. J. Pharm. 20, 119-127

18 Blumenthal, R., Weinstein, J.N., Sharrow, S.O. and Henkart, P. (1977) Proc. Natl. Acad. Sci. USA 74, 5603-5607

19 Magin, R.L. and Weinstein, J.N. (1984) in Liposome Technology (Gregoriadis, G., ed.), pp. 140-141, CRC Press, Boca Raton, FL

20 Senior, J. and Gregoriadis, G. (1984) in Liposome Technology (Gregoriadis, G., ed.), p. 266, CRC Press, Boca Raton, FL 